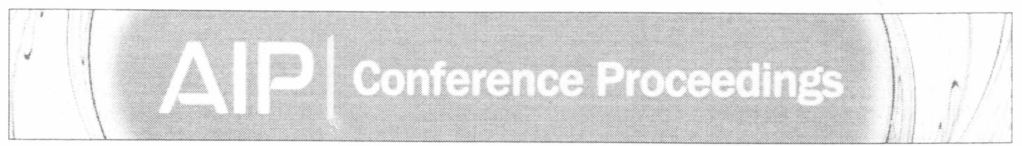

Comparative analysis of Goodwin's business cycle models

A. O. Antonova, S. Reznik, and M. D. Todorov

Citation: AlP Conference Proceedings 1773, 060002 (2016); doi: 10.1063/1.4964977

View online: http:/dx dol org/10.1063/1.4964977

View Table of Contents: http/scitation ap org/content/aip/proceeding/aipcp/1773?ver $=p \mathrm{dfcov}$

Published by the AlP Publishing

Articles you may be interested in

Dynanics of the driven Goodwin business cycle equation

AIP Conf. Proc. 1684, 050002 (2015): 10.1063/1.4934298

On periodic solutions of Goodwin's business cvcle model with onlv floor in induced investment

AIP Conf. Proc. 1561, 248 (2013); 10.1063/1.4827235

Dimensionless form for Goodwin's equation of business cycle

AlP Conf. Proc. 1487, 168 (2012); 10.1063/1.4758955

Distributed Time Delay Goodwin's Models of the Business Cvcle

AlP Conf. Proc. 1404, 333 (2011); 10.1063/1.3659936

Analysis of Types of Oscillations in Goodwin's Model of Business Cycle

AIP Conf. Proc 1301, 188 (2010); 10.1063/1.3526614 


\title{
Comparative Analysis of Goodwin's Business Cycle Models
}

\author{
A.O. Antonova ${ }^{1, a)}$, S. Reznik ${ }^{2 . b)}$ and M.D. Todorov ${ }^{3, c)}$ \\ National Aviation Universitv, I Kosmonauvta Komarova Ave., 03058 Kvis: Ukraine \\ 2Institute for Nuclear Research. National Academy of Sciences of Ukraine, 47 Prospekt Nauky, 03680. Kyiv; Ukraine \\ ${ }^{3}$ Faculty of Applied Marhemarics and Computer science. Te Imical University of Sofia. 8 Kliment Ohridski Blvd.. \\ (ex) Sojica. Bulgaria \\ "Corresponding author: anna_antonova_08@mail.ru \\ bis.reznik@voliacable.com \\ 'mod@ru-sofia.bg
}

\begin{abstract}
We compare the behavior of solutions of Goodwin's business cycle equation in the form of neutral delay ditferential cquation with fixcd delay (ND) I model) and in the form of the differential equations of 3rd, 4th and 5 th orders (ODE model s). Such ODE model's (Tavlor series expansion of NDDE in powers of $A$ ) are proposed in N. Dharmaraj and $\mathrm{K}$. Velat Velupillai [6] for investigation of the shon periodic sawthoth oscillations in NDDE. We show that the ODE: of 3 rd, 4 th and 5 th order may approximate the asymptotic behavior of only main Gondwin's mode. but not the sawthooth modes. If the order of the Taylor series expansion exceeds 5 , then the approximate ODE becomes unstable independently of time lag $A$.
\end{abstract}

Keywords: Nonlinear business cycle model, neutral delay equation. limit cycle

PACS: $02.30 . \mathrm{Hq}, 02.60 \mathrm{Cb} \mathrm{MSE}: 70 \mathrm{~K} 40,70 \mathrm{~K} 50$

\section{INTRODUCTION}

In |1] Goodwin has proposed a business cycle model in the form of the neutral delay differential equation

$$
\varepsilon \dot{y}(t)+\left(1-(t) y(t)=\varphi(t)(t-(t))+A_{0}(t)\right.
$$

Here $y$ is income, $\varphi(t)$ is induced investment, $A_{0}$ is the autonomous investment, $l$ is the time, $\varepsilon>0$ and $A>0$ are the time-lag of the multiplier and the time-lag between the investment decisions and the resulting outlays, $\alpha$ is the marginal propensity to consume, $0 \leq \alpha \leq 1$, and $y=\frac{d}{d t}$. The function $\varphi(x)$ satisfies the conditions

$$
\varphi^{\prime}(x) \geq 0, \varphi(0)=0, \varphi^{\prime}(0)=r>0, \lim _{r \rightarrow-\infty}=\varphi_{r}, \lim _{1 \rightarrow+\infty}=\varphi_{r},
$$

where $r$ is the acceleration coefticient, $\varphi$, and $\varphi$, are the Hicksian ceiling and fioor.

Goodwin has found for Eq. (1) the limit cycle with a long period $T \gg \theta$ (see also |2]). As shown in [3], |4|, [5] in addition to Goodwin's mode there exist the short periodic oscillations with periods $\theta, \theta / 2, \theta / 3$.

Strotz et al. $|4|$ has suggested that such short periodic oscillations can be obtained by using Taylor's expansion approach in powers of $\theta$ for Eq. (1). In the concluding part of [4] they predict the hypothesis: "Had Goodwin approximated his nonlinear difference-differential equation by using the first four terms of the Taylor's series expansion of the lagged function, the resulting approximating equation would have been a nonlinear differential equation of the fourth order, which we believe would have had two limit cycle solutions rather than one, both dependent on initial conditions. Improving the approximation by retaining more terms of the Taylor's expansion would increase the order of the differential equation and this would increase the number of solutions provided by the approximation." ([4], p. 406-407).

This idea has been implemented in $[6]$, in which the neutral delay differential equation (1) has been approximated by ordinary differential equations of orders 3.4 and 5. Based on the simulation of these three new models of the

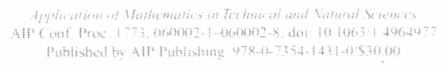

$0600002-1$ 
business cycle differential equations, the duthors concluded: "It is very interesting to see how the system behaves when more terms in the Taylor's expansion are retained. Moreover, as the orders increased, the system tended to have more number of solutions and became more sensitive to the initial conditions. Strotz et al. insightfully noted that there is not one but at least 25 difterent limit cycles as solutions for Goodwin's model, and in this exercise. by simulating the model for orders up to 5 , we have found that there are at least 45 different limit cycles, all depending upon the initial conditions" (|6], p.34). .

These results of $|6|$ seem strange to us. In this paper we reconsider the Taylor series expansion approach |4|. [6]. We obtained the analytical solution $y(t)$ for the difterential equation of third order and compared it with the analytical solution of the delay differential equation (1) [7]. We also carried out the numerical simulation of differential equations [6] of orders 4, 5 and 6 and showed that the Taylor series expansion of delay differential equation (1) is incorrect for the investigation of the short periodic solutions.

\section{ANALYTICAL AND NUMERICAL STUDIES OF THE APPROXIMATION OF DELAYED GOODWIN'S EQUATION BY TAYLOR SERIES EXPANSION}

\section{NDDE solutions}

Consider first the solutions of the delay equation (1). As in [6] we choose the following values of the parameters: $\varepsilon=0.5, \alpha=0.6, H=1, A_{0}=0, r=2, \varphi_{1}=9, \varphi_{i}=-3$. Values of $y, \varphi_{1}$ and $\varphi_{1}$ are expressed in billions of dollars per year. $t . \varepsilon, \theta$ and $r-$ in years. We choose also the piecewise-lincar function

$$
\varphi(j)= \begin{cases}\varphi_{i}, & y<y_{f}=r^{-1} \varphi_{f}, \\ r y, & y_{f}=r^{-1} \varphi_{r}>y \geq y_{l} . \\ \varphi_{i}, & j \geq y_{i} .\end{cases}
$$

This form of $\varphi(j)$ is convenient tor analytical solution of Eq. (1). For these parameters, the tixed point of Eq. (1) (0.0) is unstable and either the short-period oscillations or Goodwin's mode can be excited depending on the initial function $\Phi(t), t \leq 0$. Let $\Phi(t)=t$. Then by using the method of steps we obtain the analytical solution of Eq. (1) |71:

$$
\begin{aligned}
& y_{d_{t}}=5\left(1-e^{-0.8 t}\right) . \quad 0 \leq t<1 . \\
& y_{d_{2}}=e^{-0.8 i}(35.61 t-29.48) . \quad 1 \leq t \leq 2 \\
& y_{d_{1}}=-69.7 e^{-0) 8 t}+22.5 . \quad 2 \leq t<t_{1}=2.5375 \\
& \left.y_{d_{4}}=e^{-0.8 t} \mid-126.8 t^{2}+780.6 t-1062.6\right] . \quad t_{1} \leq t<3 \\
& y_{d_{s}}=-110.15 e^{-0.8 t}+225, \quad 3 \leq t<t_{2}=3.59321 \\
& y_{d_{b}}=e^{-0.8 t}\left(301 t^{3}-4811.3 t^{2}+23235.4 t-35047.2\right) . \quad t_{2} \leq t<t_{3}=3.7493 \\
& y_{d_{-}}=452 e^{-t) 8 t}-7.5, \quad t_{3} \leq t<4 \\
& v_{d x}=-284 e^{-0.8 t}+22.5, \quad 4 \leq t<t_{4}=4.59321 \\
& y_{d d_{t}}=e^{-0.8 i}\left(-535.92 t^{4}+16244.6 t^{3}-171087 t^{2}+752025 t-1179748 . \quad t_{4} \leq t<t_{5}=4.63\right. \\
& y_{d_{111}}=916.5 e^{-618 t}-7.5 . \quad t_{5} \leq t<5 .
\end{aligned}
$$

The dependencies $y_{d}(t)$ and $\dot{y}_{d}(t)$ are shown in Figure 1 . When $t>0$ the solution acquires clear relaxation behavior with the period $T=1$. The solution $y_{d}(t)$ includes three kinds of terms: the first corresponds to the solution of differential equation $\varepsilon y(t)+(1-\alpha) y(t)=\varphi_{i}$, the second - to $\varepsilon y(t)+(1-\alpha) y(t)=\varphi_{r}$, and the third corresponds to the differential equation with delay $\varepsilon y(t)+(1-(t) y(t)=r y(t-\theta)$. We see that the time interval $\wedge t$ associated with the time delay $\theta$ decreases dramatically: if $3<t<4, \Delta t=0.16$, and if $4<t<5, \Delta t=0.04$. For $t \gg 1$ we tind

$$
y_{d}(t) \rightarrow \begin{cases}22.5-k_{1} e^{-0.8(t)}, & 0 \leq \mid I\}<0.5982 \\ -7.5+k_{2} e^{-0.8(|t|-1)}, & 0.5982 \leq\{t \mid<1 .\end{cases}
$$

where $\{t\}=t \bmod \theta$ is the fractional part of $t, k_{1}=14.9753, k_{2}=5.0247$. The quite different functions $y_{d}(t)$ and $y(t)$ we have if $\Phi(t)=2 t, t \leq 0$ (Figure 2). This is the Goodwin mode. 

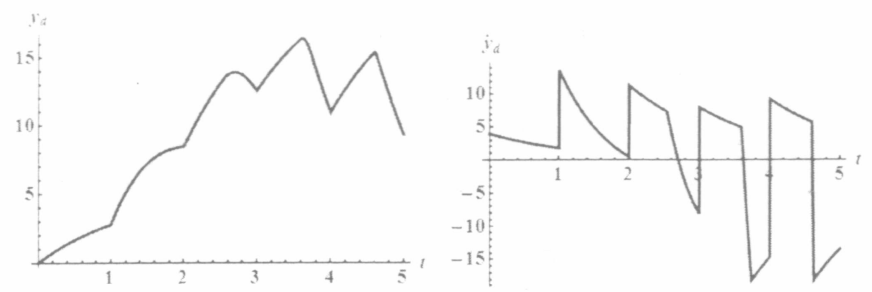

FIGURE 1. Solution of Eq. $(1) y_{d}(t)$ and $i(t)$ for initial function $\Phi(t)=t . t<0$
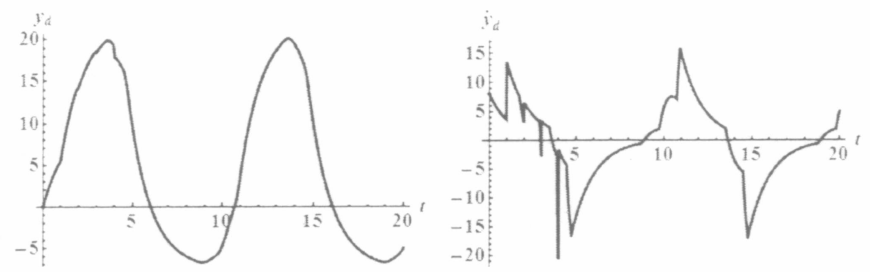

FIGURE 2. Solution of Eq. (1) $v_{d}(t)$ and $v_{d}(t)$ for initial function $\Phi(t)=2 t, t \leq 0$

Remark. By the numerical modeling of Eq. (1) with the piecewise-linear function $\varphi(j)$ and $\varepsilon=0.5, \alpha=0.6$. $H=1, A_{0}=0, r=2, \varphi_{c}=9, \varphi_{f}=-3$ we can find that for initial function $\Phi(t)=a t, t \leq 0$ with $a \in(-0.65,0) ;(0,1.79)$ the sawtooth oscillations with period 1 arise. For a outside this interval Goodwin's mode with period 10 excites.

\section{Taylor's series expansion solutions}

Now we investigate the possibility of the existence of short- and long periodic oscillations in the differential equations [6]. Following $\mid 1,6]$ we write Eq. (1) in the form

$$
\varepsilon y(\tau+\theta)+(1-\alpha) y(\tau+\theta)=\varphi(y(\tau))+A_{0}(\tau+\theta) . \quad \tau \geq-\theta, \quad \tau=t-\theta .
$$

By Taylor's series expansion of $y(\tau+\theta)$ and $y(\tau+\theta)$ at $\theta=0$ in powers of $\theta$ we obtain from Eq. (3) such differential equations $[6]$ :

$$
L_{n}(D) v_{n}(\tau)-\varphi\left(\dot{y}_{n}(\tau)\right)=0,
$$

where $L_{n}(D)$ are the linear differential operators with constant coefficients,

$$
L_{n}(D)=(\varepsilon D+1-\alpha)\left(1+\theta D+\frac{(\theta D)^{2}}{2 !}+\ldots+\frac{(\theta D)^{n-1}}{(n-1) !}\right), \quad D=\frac{d}{d \tau} .
$$

For simplicity, in Eqs. (4) the autonomous investment $A_{0}$ is skipped. If $\varphi(y)$ is the piecewise linear function, each of the equations (4) can be written as follows

$$
\left\{\begin{array}{lll}
L_{n}(D) y_{n}(\tau)=\varphi_{f}, & \dot{y}_{n}(\tau)<\dot{y}_{f} . & (a) \\
\left(L_{n}(D)-r D\right) y_{n}(\tau)=0 . & \dot{y}_{c}>\dot{y}_{n}(\tau) \geq \dot{y}_{f}, & (\text { b) } \\
L_{n}(D) y_{n}(\tau)=\varphi_{i} . & y(\tau) \geq \dot{y}_{c} . & (c)
\end{array}\right.
$$

The general solution of Eqs. (5a) and $(5 \mathrm{c})$ may be written as a superposition of inhomogeneous solutions $\varphi_{f}(1-\alpha)^{-1}$. $\varphi_{c}(1-\alpha)^{1}$ and homogeneous solutions. For homogeneous ODE $(5 \mathrm{a})$ and $(5 \mathrm{c})$ we substitute $y_{n}=e^{t r}$, and obtain 
the characteristic cquations $L_{n}(t)=0$. For Eq. (5b) we substitute $y_{n}=e^{\prime \prime}$ and obtain the characteristic cquations $L_{n}(\mu)-r \mu=0$.

Note that, the characteristic polynomials $L_{n}(\lambda)$ can be written as

$$
L_{n}(1)=\left(1-\psi+(\varepsilon) P_{n} \mid(q) . \quad q=i \theta . \quad P_{n}(q)=1+q+\frac{q^{2}}{2}+\ldots+\frac{q^{n-1}}{(n-1) !}\right.
$$

Therefore, all cquations $L_{n}(\lambda)=0$ have the root $t_{1}=-(1-\alpha) \varepsilon^{-1}$. which corresponds to the damped solution. Although, all coefficients of the polynomials $P_{n-1}(\lambda)$ are positive, they are the Hurwitz polynomials $\left(i . e_{\text {.. all }} \mathrm{Re} \lambda_{h}<0\right)$ only for $n=2,3,4$ and 5 . We see that starting with $n=6$ at least one eigenvalue $\lambda_{k}$ has positive real part. This means that the Taylor expansion in powers of time delay $\theta$ is incorrect. since the differential equation $(5)$ gives rise to a non-physical infinitely growing solutions that are ahsent in the delay differential equation (1). Therefore, this method should never be used for $n \geq 6$.

Consider Eqs. (5) with $n=2,3,4$ and 5 . For selected values of parameters the roots $\mu_{k}$ and $i_{k}$ (except root $l_{1}=-0.08$ ) are shown in Table 1 and Table 2.

\begin{tabular}{|c|c|c|c|c|c|c|}
\hline \multicolumn{4}{|c|}{ TABLE $1 . \mu_{k}$ roots } & \multicolumn{3}{|c|}{ TABLE $2 . \lambda_{k}$ roots } \\
\hline$n$ & & & & $n$ & & \\
\hline 2 & 0.46 & 1.74 & & 2 & -1 & \\
\hline 3 & -4 & $0.6 \pm 0.2 i$ & & 3 & $-1 \pm i$ & \\
\hline 4 & $-2.45 \pm 2.73 \mathrm{i}$ & $0.554 \pm 0.22 \mathrm{i}$ & & 4 & -1.6 & $-0.70 \pm 1.81 \mathrm{i}$ \\
\hline 5 & -4 & $0.548 \pm 0.22 i$ & $-0.95 \pm 3.58 \mathrm{i}$ & 5 & $-1.73 \pm 0.89 i$ & $-0.27 \pm 2.5 \mathrm{i}$ \\
\hline
\end{tabular}

Table 1 shows that for all $n=2,3,4$ and 5 the stationary point $(0,0, \ldots, 0)$ is unstable. Therefore, the nonlinear oscillations of income due to the induced investment will be excited.

Now we find the analytic solution of differential equation of third order (5). We do that by using the CAS package Mathematica 8. For piecewise-linear investment function this equation can be written as

$$
\begin{array}{rcc}
5 \dddot{y}+14 y+18 j+8 y=180 . & y \geq 4.5 . & \text { (a) } \\
5 y+14 j-22 y+8 y=0 . & -1.5 \leq i<4.5 . & \text { (b) } \\
5 \dddot{y}+14 j+18 j+8 y=-60 . & y<1.5 . & \text { (c) }
\end{array}
$$

For the initial function $\Phi(t)=t$ or $\Phi(\tau)=\tau+\theta=\tau+1$ the initial conditions for $y(\tau)$. $y(\tau)$ and if $\tau)$ can be set as

$$
y(-1)=\Phi(-1)=0 . \quad y(-1)=\Phi(-1)=1 . \quad y(-1)=\Phi(-1)=0 .
$$

Since $y(-1)=1<y_{i}=4.5$. we solve first Eys. (6b) and $(7)$ with stopping condition $y\left(\tau_{1}\right)=4.5$. Using software package Mathematica we obtain $\tau_{1}=0.820855$, and

$$
y 1=\frac{1}{53}\left(-3 e^{-4(1+\tau)}+\left(196 \sin \frac{1+\tau}{5}+\cos \frac{1+\tau}{5}\right) e^{0(x)+r)}\right) . \quad-1 \leq \tau \leq \tau_{1} .
$$

Since $v\left(\tau_{1}\right)=v_{i}=4.5$, for $\tau>\tau_{1}$ we have to solve Eq. $(6 \mathrm{a})$ with initial conditions

$$
y 2\left(\tau_{1}\right)=y 1\left(\tau_{1}\right), \quad \dot{y} 2\left(\tau_{1}\right)=\dot{y} l\left(\tau_{1}\right) . \quad \dot{y} 2\left(\tau_{1}\right)=\dot{y} 1\left(\tau_{1}\right)
$$

with stopping condition $\mathrm{v}\left(\tau_{2}\right)=4.5$. We obtain $\tau_{2}=3.02331$, and

$$
y_{2}=22.5-44.6209 e^{-0.8 \tau}+(-6.52918 \sin \tau+22.7598 \cos \tau) e^{-\tau} . \quad \tau_{1} \leq \tau \leq \tau_{2} .
$$

For $\tau>\tau_{2}$ we solve Eq. (6b) with initial conditions

$$
y 3\left(\tau_{2}\right)=y 2\left(\tau_{2}\right), \quad y 3\left(\tau_{2}\right)=y 2\left(\tau_{2}\right), \quad y 3=\ddot{y} 2\left(\tau_{2}\right)
$$


with stopping condition if $\left.t_{3}\right)=i_{1}=-1.5$. We obtain $t_{i}=4.13922$.

$$
13=-11429.3 e^{-t+}+\left(5.22635 \cos \frac{T}{5}-2.55877 \sin \frac{T}{5}\right) e^{06 \tau} \cdot \tau_{2} \leq T \leq T_{3}
$$

Continuing the procedure in the same way we get

$$
\begin{gathered}
y 4=-7.5+1103.2 e^{-0) 8 t}+(78.9118 \sin \tau+1375.78 \cos \tau) e^{-\tau} . \quad \tau_{5} \leq \tau<\tau_{4}=8.2027, \\
y 5=9.22329 \cdot 10^{12} e^{-4 \tau}-\left(0.0829994 \cos \frac{\tau}{5}+0.0503717 \sin \frac{\tau}{5}\right) e^{0.6 \tau} . \quad \tau_{4} \leq \tau<\tau_{5}=9.92723, \\
16=22.5-103909 e^{08 \tau}+(183997 \sin \tau-324412 \cos \tau) e^{t} . \quad \tau_{5} \leq \tau<12.78 .
\end{gathered}
$$

The obtained dependencies $y(t)$ and $y(t)$ are shown on Figure 3. We see that the differential equations (6) do not describe even qualitatively the relaxation oscillations with period $\theta$. Eqs. (6) describe only the excitation of the Goodwin mode. For the initial function $\Phi(t)=2 t, t \leq 0$ or $\Phi(\tau)=2(\tau+\theta)$ the initial conditions for $y$ can be set as
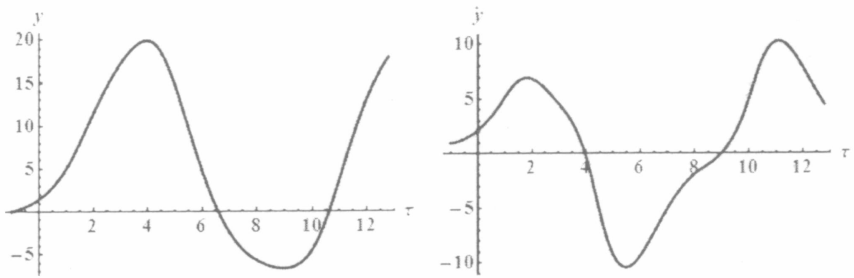

FIGURE 3. Dependencies $x(t)$ and $i(t)$ for Eq. (6) with initial conditions $y(-1)=(), y(-1)=1, y(-1)=(0$
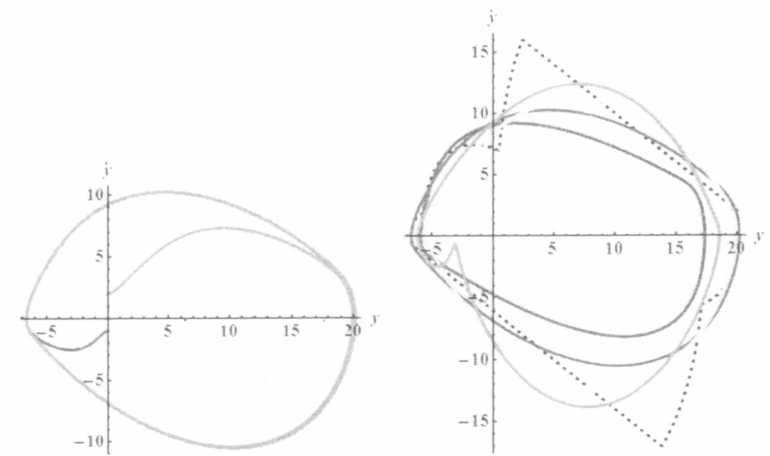

FIGURE 4. Phase curves iv(v): a) Eq. (6) with the initial function $\Phi(\mathrm{r})=a(\mathrm{r}+\theta)$ : blue: $a=-1$, green: $a=1$, red: $a=2 ;$ b) Limit cycles for Eq. (5): blue: $n=2$, purple: $n=3$, cyan: $n=4$, red: $n=5$; dashed line - Eq. (1)

$$
y(-1)=0, \quad \dot{y}(-1)=2, \quad \dot{y}(-1)=0 .
$$


In this case only the initial stage of the functions $y t)$ and $1 t t)$ will change, but for $t \gg 1$ the phase trajectory tends to the same Goodwin's mode (sec Figure 4a).

We carried out the numerical modeling of Eqs, (6) with the different initial conditions and for $t \gg 1$ we observed only the Goodwin mode. For Eqs. (5) with $n=4$ we observed also only the Goodwin mode (they are slightly different in period and peak-to-peak amplitude): for $n=5$ there arose the distortion of the shape of the Goodwin limit cycle (see Figure $4 \mathrm{~b}$ and Figure 5). Finally, when $n=6$ we have seen nonphysical exponentially growing solution (sec Figure 5).
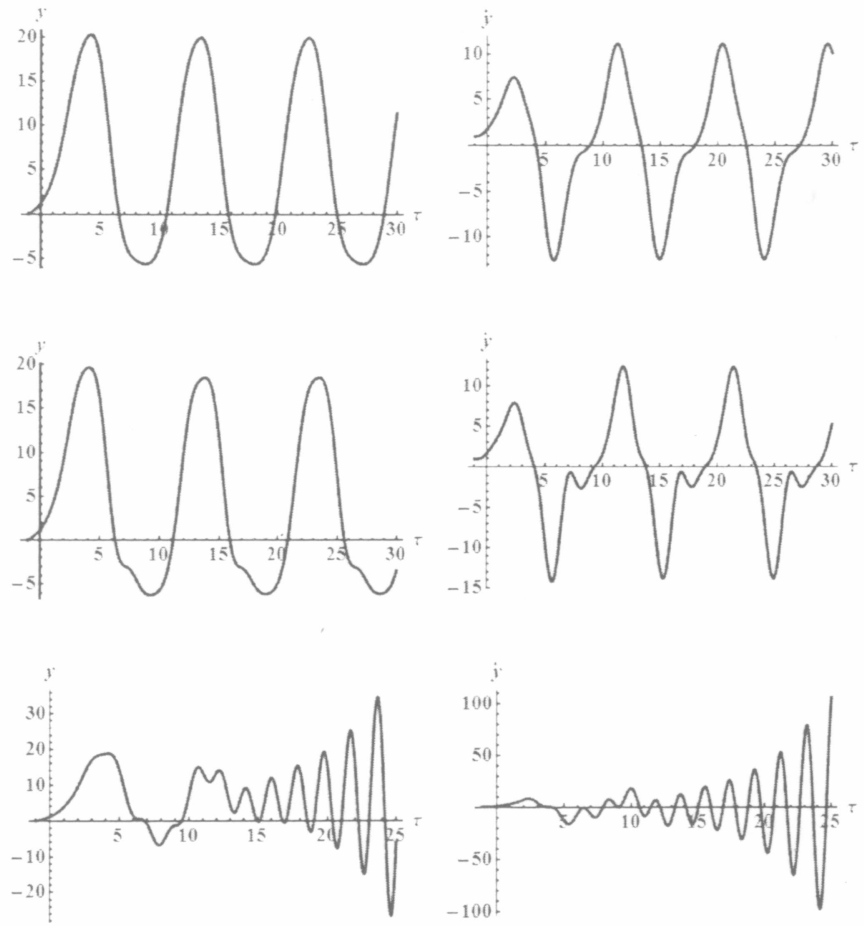

FIGURE 5. The solutions $v(t)$ and $i(t)$ of Eq. (5) for $n=4, n=5$ and $n=6$

\section{DISCUSSION AND CONCLUSIONS}

Our analysis shows that the use of a Taylor series expansion approach for delay differential equation (1) is questionable. Firstly, it produces ODE's with non-physical solutions which grow with time. Eq. (1) does not have such solutions. Secondly, the ODE models of $3 \mathrm{rd}, 4$ th and 5 th orders do not contain the short-periodic solutions, which 
are typical for the neutral delay equation (1). Thirdly. all of these models poorly modeled even the properties of Goodwin's mode as shown in Figure 4 b.

The correct approach to the modeling of Goodwin's mode with the fixed delay hy the ODF equations has heen proposed by Matsumoto [8]. This model has the form of the integro-differential equation

$$
\varepsilon y(t)+(1-\alpha) y(t)=\varphi(z(t))+A_{t} . \quad z(t)=\int^{\prime} w(t-s, H) y(s) d s
$$

where the weight function $w$ is the $\Gamma$-distribution

$$
w=w(s, \theta)=\frac{e^{\frac{i}{4}}}{\theta(k-1) !}\left(\frac{k s}{\theta}\right)^{k} . \quad k=1,2, \ldots
$$

Eq. (8) contains two parameters $A$ and $k$, on which the average delay time $T_{d}$ and its variance $\sigma_{d}^{2}$ depend

$$
T_{d}=\int_{0}^{\infty} s w_{k}(s, \theta) d s=\frac{k+1}{k} \theta, \quad \sigma_{d}^{2}=\int_{0}^{\infty}\left(s-T_{d}\right)^{2} w_{k}(s \cdot \theta) d s=\frac{k+1}{k^{2}} \theta^{2} . \quad \frac{v_{d}}{T_{d}}=\frac{1}{\sqrt{k+1}} .
$$

If $k \rightarrow \infty$, the distribution $w_{k}(s . \theta)$ tends to Dirac's delta function $\partial(s-\theta)$ and Eq. (8) reduces to Eq. (1). It can be shown that Eq. (8) is equivalent to the system of differential equations

$$
\left\{\begin{array}{l}
\varepsilon D y(t)+(1-\alpha) y(t)=\varphi(s(t))+A_{t)} \\
\left(1+\frac{\theta}{k} D\right)^{k+1} z(t)=D y(t) .
\end{array} \quad k=1,2, \ldots\right.
$$

If $\varphi(y)$ is the piecewise linear function, the equations $(9)$ can be written as follows

$$
\begin{array}{clc}
M_{k}(D) z_{k}(t)=0 . & z_{k}(t)<j_{t} & (a) \\
\left(M_{k}(D)-r D\right) z_{k}(t)=0 . & j_{c}>z_{k}(t) \geq y_{t} & (b) \\
M_{k}(D) z_{k}(t)=0 . & z_{k}(t) \geq j_{i} & \text { (c) }
\end{array}
$$

$\operatorname{Herc} M_{k}(D)=(\varepsilon D+1-\alpha)\left(1+\frac{u}{k}\right)^{k+1}$. Note that all roots of characteristic equations $M_{k}(\lambda)=0$ are negative.

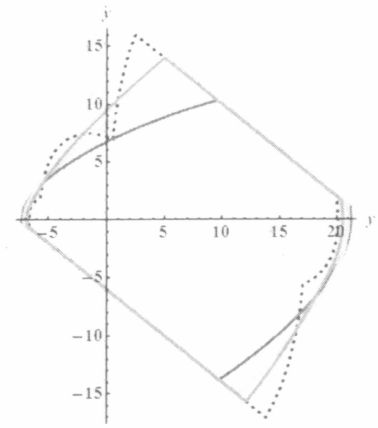

FIGURE 6. Phase curves $v(y)$ for Eq. (10): blue: $k=1$, green: $k=2$, red: $k=6$, dashed line - for Eq. (1)

Even for $k \geq 2$, Eq. (10) correctly describes the behavior of the Goodwin mode (see Figure 6). But the Matsumoto approach is not very effective to describe the short periodic oscillations. As shown in [9] the short periodic oscillations 
exist only for very narrow delay distribution when the variance of the delay distribution much less than the average delay.

$$
\gamma_{c r}=\frac{\sigma_{d}}{T_{d}} \ll 1 .
$$

For $\varepsilon=0.5, \alpha=0.6, \theta=1, r=2, \varphi_{i}=9, \varphi_{1}=3$ the piecewise linear investment function. we find that $\gamma \approx 0.1$. This means that one necds to use the Matsumoto model with $k>99$. Apparently it is morc preferably to use the following delay equation $|9|$

$$
\varepsilon y(t)+(1-\alpha) y(t)=\varphi\left\{\frac{1}{2 \delta}[y(t-H+\delta)-y(s-A-\delta)]\right\}
$$

Eq. $(11)$ is derived under the assumption that $w(s)$ is symmetrically distributed around a mean value $\theta$ between the minimal $A-\delta$ and maximal $A+\delta$ delays, $\delta \ll H$ is the half width of the distribution.

$$
w(s)=\frac{1}{2 \delta}[H(s-\theta+\delta)-H(s-\theta-\delta) \mid .
$$

where $H(s)$ is the Heaviside function.

\section{REFERENCES}

11) R. M. Goodwin (1951) tecotzestetities 19.1-17.

121 R. G. D. Allen. Macro-Economic Theorv: A Mathematical Treatment (Macmillan, London, 1968), pp.379382.

131 F. E. Bothwell (1952) Exwomiet 20. 269-284

14] R. H. Strotz. J.C. McAnulty, and J.B. Naines (1953) temismente it 21. 390-411.

151 A. O. Antonova, S. N. Reznik, and M. D. Todorov, "Analysis of types of oscillations in Goodwin's model of business cycle," in Application of Mathematics in Technical and Natural Sciences (AMiTaNS' 10). AIP Conference Procedings Vol. 1301, edited by M. D. Todorov and C.I. Christov (American Institute of Physics, Melville, NY, 2010). pp. 188-95.

[6] N. Dharmaraj and V.K. Velupillat. The Time-to-Build Tradition in Business Cycle Modelling. Algorithmic Social Sciences Research Unit, Discussion Paper Series, 11-09 (2011), http://www. assru.economia. unitn.it/files/DP\_09\_2011.pdf

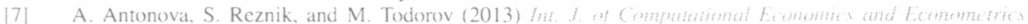
3( $3 / 4), 146-163$.

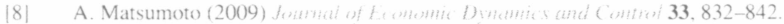

[9] A.O. Antonova, S.N. Reznik and M.D. Todorov, "Distributed time delay Goodwin's models of the business cycle," in Application of Mathematics in Technical and Natural Sciences (AMiTaNS"ll). AIP Conference Procecdings Vol.1404, edited by M. D. Todorov and C.I. Christov (American Institute of Physics, Melville. NY. 2011), pp.333-339 